\title{
Espalhamento e tensão superficial de gotas de soluções com herbicidas e adjuvantes em folhas de Digitaria insularis
}

\author{
Adriana Cologni Salvalaggio ${ }^{1}$, Neumárcio Vilanova da Costa ${ }^{1}$, Victor Natan Cazzo ${ }^{1}$, Elio \\ Conradi Junior ${ }^{1}$, Alesson Felipe Eckert ${ }^{1}$
}

\begin{abstract}
${ }^{1}$ Universidade Estadual do Oeste do Paraná, campus de Marechal Cândido Rondon, Marechal Cândido Rondon, Paraná, Brasil. E-mail: adriana.salvalaggio@outlook.com,neumarciovc@ hotmail.com, victtornattan@ hotmail.com, transconradi@hotmail.com, alessonfelipe@ hotmail.com.
\end{abstract}

Recebido: 31/05/2017; Aceito: 02/05/2018.

\begin{abstract}
RESUMO
Objetivou-se avaliar a tensão superficial e eficiência do espalhamento de gotas de soluções com herbicidas e adjuvantes em diferentes concentrações em folhas de Digitaria insularis. O delineamento experimental utilizado foi inteiramente casualizado, com quatro repetições. Os tratamentos utilizados foram glyphosate (Roundup Original ${ }^{\circledR}$, $360 \mathrm{~g} \mathrm{~L}^{-1}$ ) na dose de $960 \mathrm{~g} \mathrm{~L}^{-1}$ e glufosinato-sal de amônio (Finale ${ }^{\circledR}, 200 \mathrm{~g} \mathrm{~L}^{-1}$ ), na dose de $400 \mathrm{~g} \mathrm{~L}^{-1}$ com adição dos adjuvantes: óleo mineral $\left(\right.$ Nimbus $\left.^{\circledR}\right)$, óleo vegetal $\left(\right.$ Aureo $\left.^{\circledR}\right)$ e glicerina $\left(\right.$ Hygrogem $^{\circledR}$, subproduto do biodiesel de soja), nas concentrações de: 0,$00 ; 0,08 ; 0,16 ; 0,31 ; 0,63 ; 1,25 ; 2,50$ e 5,00\% (v/v), em folhas de Digitaria insularis. $\mathrm{O}$ espalhamento das gotas das soluções foram avaliadas nas faces foliares adaxial e abaxial. A eficiência no espalhamento de gotas nas folhas de $D$. insularis foi dependente do herbicida da face foliar, bem como da concentração dos adjuvantes avaliados. Observou-se que os adjuvantes foram eficientes em reduzir a tensão superficial das soluções com os herbicidas, com exceção para a glicerina adicionada ao glifosato. O glifosato+óleo mineral $(3,2 \%)$ e o glufosinato+óleo vegetal $(0,3 \%)$ foram os mais eficientes no espalhamento na face adaxial da folha de $D$. insularis, enquanto que o glifosato+óleo mineral $(0,6 \%)$ e o glufosinato+óleo mineral $(1,5 \%)$ foram os mais eficientes no espalhamento na face abaxial.
\end{abstract}

Palavras-chave: Plantas daninhas, Capim-amargoso, Tecnologia de aplicação.

\section{Surface spreading and tension of drops of solutions with herbicides and adjuvants in Digitaria insularis leaves}

\begin{abstract}
This project aimed to evaluate the surface tension and efficiency of spreading drops of solutions with herbicides and adjuvants at different concentrations in Digitaria insularis leaves. The experimental design was completely randomized, with four replications. The treatments were glyphosate (Roundup Original ${ }^{\circledR}, 360 \mathrm{~g} \mathrm{~L}^{-1}$ ) and glufosinate ammonium salt $\left(\right.$ Finale $^{\circledR}, 200 \mathrm{~g} \mathrm{~L}^{-1}$ ), with the addition of adjuvants: mineral oil (Nimbus ${ }^{\circledR}$ ), vegetable oil (Aureo ${ }^{\circledR}$ ) and glicerine (Hygrogem ${ }^{\circledR}$ byproduct from the soybean biodiesel) at concentrations of 0,$00 ; 0,08 ; 0,16 ; 0,31 ; 0,63$; 1,$25 ; 2,50$ e $5,00 \%(\mathrm{v} / \mathrm{v})$, in Digitaria insularis leaves. The droplet spreading of the solutions were evaluated in the adaxial and abaxial foliar faces. The efficiency of droplet spreading on leaves of $D$. insularis was dependent on the foliar herbicide as well as the concentration of adjuvants evaluated. It was observed that the adjuvants were effective in reducing the surface tension of the solutions with the herbicides, except for the glycerin added to the glyphosate. Glyphosate + mineral oil $(3,2 \%)$ and glufosinate + vegetable oil $(0,3 \%)$ were the most efficient in spreading on the adaxial side of $D$. insularis leaf, whereas Glyphosate + mineral oil $(0,6 \%)$ and glufosinate + mineral oil $(1,5 \%)$ were the most efficient in the abaxial face scattering.
\end{abstract}

Keywords: Weeds, Sourgrass, Application technology. 


\section{Introdução}

A Digitaria insularis, popularmente conhecida como capim-amargoso, ocorre em regiões tropicais e subtropicais da América, geralmente infestando pastagens e sistemas de cultivo (Machado et al., 2008). As plantas daninhas em geral competem com as culturas por água, luz e nutrientes resultando na diminuição do rendimento e na qualidade do produto, além do mais, podem aumentar o custo de produção, já que são necessárias estratégias de controle diferenciadas nas lavouras (Galon et al., 2007; Vidal et al., 2004; Voll et al., 2002). Dentre as técnicas de controle de plantas daninhas, a principal estratégia de manejo tem sido aplicações de herbicidas devido à praticidade, rapidez e eficiência. $\mathrm{O}$ controle pode ser realizado com produtos sistêmicos ou de contato, aplicados na dessecação, pré e pós-emergência, isolados ou em mistura, e também aplicações sequenciais do mesmo ou de diferentes herbicidas (Procópio et al., 2006).

Neste contexto, destaca-se que o glyphosate tem sido um dos herbicidas mais utilizados para o controle de plantas daninhas em diversos sistemas de produção, devido ao seu amplo espectro de ação e registro para diversas culturas. Porém, o capim-amargoso tem apresentado resistência ao herbicida glyphosate, o que torna seu controle mais difícil. De acordo com Machado et al. (2006), em áreas onde há uso contínuo do herbicida glyphosate, tem-se constatado que plantas mais desenvolvidas e com rizomas, seu controle tornase ineficiente.

Esta ocorrência de resistência ao controle pode ser explicada devido à intensa utilização do glyphosate, que favorece o aumento da pressão de seleção e juntamente com a adaptação das plantas ao ecossistema e a sistemas conservacionistas de manejo do solo, acabam contribuindo para a seleção de biótipos de plantas resistentes (Moreira et al., 2007). Devido ao aparecimento de resistência das plantas daninhas a algumas moléculas, dentre elas a do glyphosate, tem-se a preocupação por rotacionar as moléculas e modo de ação dos herbicidas nas aplicações.

Desse modo, tem-se utilizado outros herbicidas como o glufosinato sal de amônio em aplicações isoladas, em mistura ou sequencias para o controle de D. insularis (Melo et al., 2012). O uso de tecnologias de aplicação também tem sido utilizado com o intuito de melhorar a qualidade da aplicação e controle das plantas daninhas. A eficiência da aplicação pode ser determinada pela adequada distribuição do produto fitossanitário no alvo e para isso, utilizam-se produtos que podem melhorar a eficiência biológica dos herbicidas e alterar as características físicas e químicas: os adjuvantes. (Cunha et. al, 2010).

Dentre as características físicas e químicas, ressaltase que a tensão superficial é uma propriedade importante durante a aplicação de herbicidas, pois uma boa molhabilidade e penetração na massa de folhas favorecem a adesividade, retenção e absorção dos produtos pelas plantas (Cunha e Alves, 2009; Baio et al., 2015). O óleo mineral e vegetal são exemplos de adjuvantes, e além destes, recentemente tem se usado também a glicerina, subproduto do biodiesel de soja.

Desta maneira, acredita-se que área de espalhamento de gotas de soluções com glyphosate e glufosinato sal de amônio nas faces foliares de $D$. insularis pode ser dependente da utilização de adjuvantes. Portanto, objetivou-se avaliar o espalhamento de soluções de glifosato e glufosinato com diferentes concentrações de adjuvantes na superfície abaxial e adaxial de folhas de D. insularis.

\section{Material e Métodos}

O delineamento experimental utilizado foi o inteiramente casualizado com quatro repetições. Os tratamentos foram compostos por soluções de glyphosate (Roundup ${ }^{\circledR}, 360 \mathrm{~g} \mathrm{~L}^{-1}$ ) na dose de $960 \mathrm{~g} \mathrm{~L}^{-1} \mathrm{e}$ glufosinato-sal de amônio (Finale ${ }^{\circledR}, 200 \mathrm{~g} \mathrm{~L}^{-1}$ ), na dose de $400 \mathrm{~g} \mathrm{~L}^{-1}$, com adição dos adjuvantes óleo mineral parafínico (Nimbus ${ }^{\circledR}$ ), óleo vegetal- éter metílico óleo de soja (Aureo ${ }^{\circledR}$ ) e um subproduto derivado da extração do biodiesel a base de glicerina-propano-1,2,3-triol $\left(\right.$ Hygrogem $\left.^{\circledR}\right)$, todos nas concentrações de: 0,00; 0,08; 0,$16 ; 0,31 ; 0,63 ; 1,25 ; 2,50$ e $5,00 \%$ (v/v).

As soluções foram preparadas com água destilada e foram acondicionadas em balões volumétricos de 1 litro. Para determinação da tensão superficial das soluções, utilizou-se a metodologia proposta por (Mendonça et al., 1999). Para cada solução foi determinado o peso (g) de 15 gotas, sendo considerada como uma repetição e foram utilizados os dados médios de quatro repetições. A tensão superficial das gotas das soluções foi determinada pela Equação: TS= P1x(72,6/P2), em que: $\mathrm{TS}=$ tensão superficial $\left(\mathrm{mN} \mathrm{m}^{-1}\right) ; \mathrm{P} 1=$ peso em gramas das gotas das soluções tratamentos; $\mathrm{P} 2$ = peso em gramas das gotas da água destilada. Considerou-se para efeito de cálculo a tensão superficial da água igual a $72,6 \mathrm{mN} \mathrm{m}^{-1}$.

As plantas de Digitaria insularis foram coletadas em condições de campo. No laboratório, as plantas foram mantidas com as raízes imersas na água para que as folhas não perdessem a turgescência.

A área de molhamento foi determinada nas faces foliares adaxial e abaxial da primeira folha logo abaixo da folha bandeira de perfilhos com inflorescências das plantas.

A temperatura ambiente do laboratório foi de $21{ }^{\circ} \mathrm{C}$ $\left( \pm 2^{\circ} \mathrm{C}\right)$ e umidade relativa do ar entre 50 e $60 \%$, monitoradas a cada hora por um termohigrômetro modelo MTH-1362W (Minipa). 
Para fixação das folhas de capim-amargoso utilizouse uma fita adesiva dupla face em uma folha de E.V.A. (Etileno Acetato de Vinila) preta e opaca, no tamanho A4. Posteriormente com o auxílio de uma micropipeta calibrada para um volume de $12 \mu \mathrm{L}$, foram aplicadas gotas das soluções nas faces foliares. Logo após a deposição, a gota foi fotografada com o auxílio de máquina fotográfica digital (Samsung ${ }^{\circledR}$ HD ST77 com 16,1 mega-pixels).

Para as análises das imagens, utilizou-se o software Quant V 1.0, calibrado por meio de uma escala com comprimento conhecido de um centímetro, desenhada na folha de E.V.A., utilizada como ponto de referência no campo de imagem para determinação da área de cores pré-definidas ocupada pela gota e em seguida transformou-se em $\mathrm{cm}^{2}$.

Os dados foram ajustados a modelos de regressão, sendo as equações escolhidas com base nos modelos significativos $(P \leq 0,05)$, normalidade e elevado $\mathrm{R}^{2}$.

\section{RESULTADOS E DISCUSSÃO}

Na Figura 1, estão apresentados os valores da tensão superficial das soluções de glifosato e glufosinato com diferentes concentrações de adjuvantes. Verificou-se nas soluções contendo glifosato que a adição tanto do óleo mineral, quanto do óleo vegetal a $1 \%$ foram eficientes em reduzir a tensão superficial em 17,6 e 21,2\% respectivamente, em relação à solução de glifosato sem adjuvante (Figura 1A). Porém, em concentrações maiores os adjuvantes apresentaram comportamentos semelhantes. Para as soluções de glifosato com glicerina, observou-se que o adjuvante não reduziu a tensão superficial à medida que se aumentou a concentração.

As maiores reduções da tensão superficial para o glufosinato + óleo mineral $(18,5 \%)$ e glufosinato + óleo vegetal $(26,6 \%)$ foram obtidas a partir da concentração $1 \%$, com tendência de estabilização com o aumento da concentração dos adjuvantes, conforme também foi verificado para o glifosato (Figura 1B). Contudo, a glicerina promoveu redução de $23,2 \%$ já a partir de $0,1 \%$. Estes resultados mostram a ocorrência de interações entre os herbicidas e adjuvantes adicionados à calda de pulverização na redução da tensão. superficial da gota, o que pode refletir na maior eficiência do produto.

Cunha et al. (2010) avaliaram a eficiência de adjuvantes sem a adição de herbicidas e verificaram que o óleo vegetal (Áureo ${ }^{\circledR}-0,25 \%$ ) promoveu tensão superficial $9,0 \%$ menor que o óleo mineral (Nimbus ${ }^{\circledR}$ $0,5 \%$ ). Estes resultados confirmam os reportados por Mendonça et al. (2007) e confirmam os obtidos no presente estudo de que o óleo vegetal tende a ser mais eficiente que o óleo mineral em reduzir tensão superficial da gota.
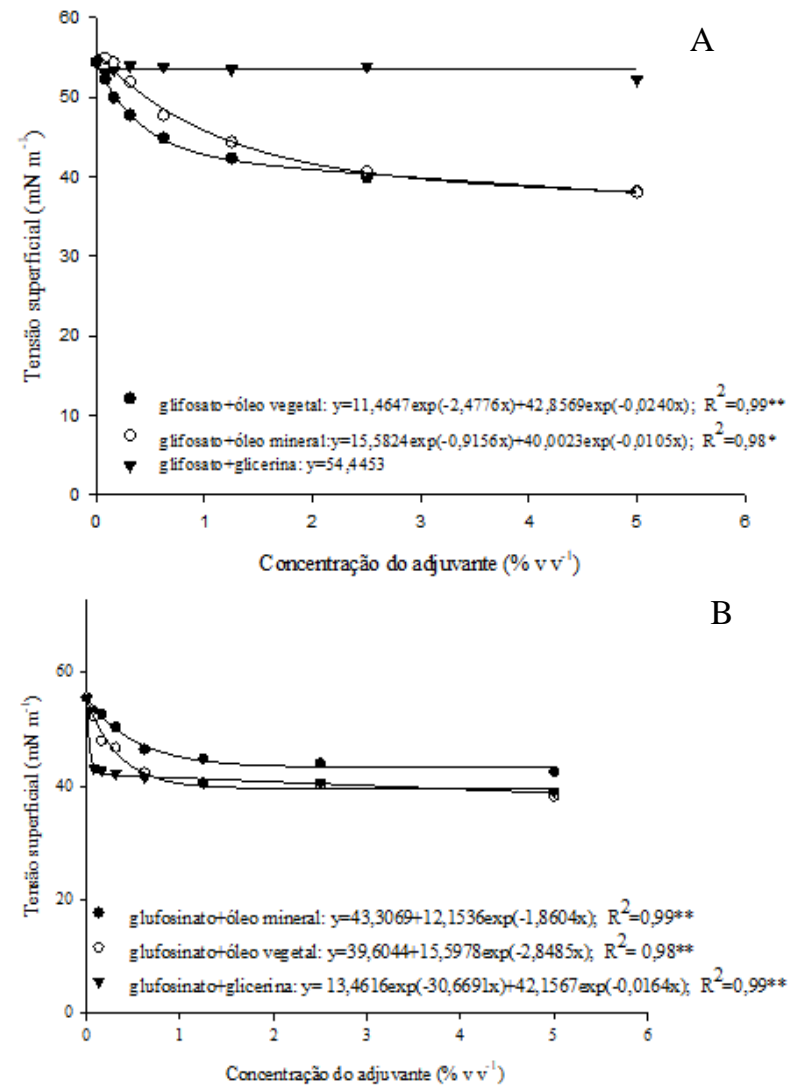

Figura 1. Tensão superficial das soluções de glifosato (A) e glufosinato (B) com diferentes concentrações de adjuvantes.

Na Figura 2, encontram-se os valores de área de espalhamento de gotas na face abaxial e adaxial da folha de $D$. insularis das soluções de glifosato com concentrações de adjuvantes. Verificou-se para a face abaxial (Figura 2A), que as soluções de glifosato+óleo mineral $(0,6 \%)$ e glifosato+óleo vegetal $(2,5 \%)$ proporcionaram 50,5 e $47,8 \%$, respectivamente, mais espalhamento do que o herbicida sem adjuvante.

O glifosato+óleo mineral $(3,2 \%)$ apresentou o máximo de espalhamento na face adaxial, sendo $43,4 \%$ superior ao herbicida sem adjuvante (Figura 2B). Entretanto, os dados de espalhamento de gotas do glifosato+óleo vegetal apresentaram ajuste linear, em que se pode verificar que para cada aumento da concentração do adjuvante ocorre espalhamento de $0,0319 \mathrm{~cm}^{2}$ na folha.

Os dados de glicerina não apresentaram ajuste para ambas as faces foliares, além de que, a glicerina apresentou menor eficiência em promover espalhamento da gota, em comparação ao óleo mineral e vegetal.

Segundo Costa et al. (2014) a adição do óleo mineral (Joint oil $^{\circledR}$ ) ao glifosato (960 $\mathrm{g} \mathrm{ha}^{-1}$ ) apresentou maior eficiência no espalhamento de gotas nas folhas de buva (Conyza bonariensis) a até a concentração de $0,75 \%$, principalmente para a face abaxial, enquanto que a adição do adjuvante não iônico (Energic ${ }^{\circledR}$ ) ao glifosato não influenciou no espalhamento da gota em ambas as faces. Os autores ressaltaram ainda que a eficiência dos 
adjuvantes no espalhamento das gotas nas faces foliares foi dependente da concentração e do herbicida, conforme verificado no presente estudo.

Mendonça et al. (1999) verificaram que o espalhamento de gotas de glifosato $\left(1440 \mathrm{~g} \mathrm{ha}^{-1}\right)$ na face adaxial de Cypeus rotundus foi 51,5; 70,8 e 93,4\% superior quando adicionou-se na calda $1 \%$ dos adjuvantes Extravon ${ }^{\circledR}$ (espalhante adesivo), Aterbane ${ }^{\circledR}$ (espalhante adesivo) e Silwet L-7 ${ }^{\circledR}$ (organosiliconado), respectivamente, em relação ao glifosato sem adjuvante.
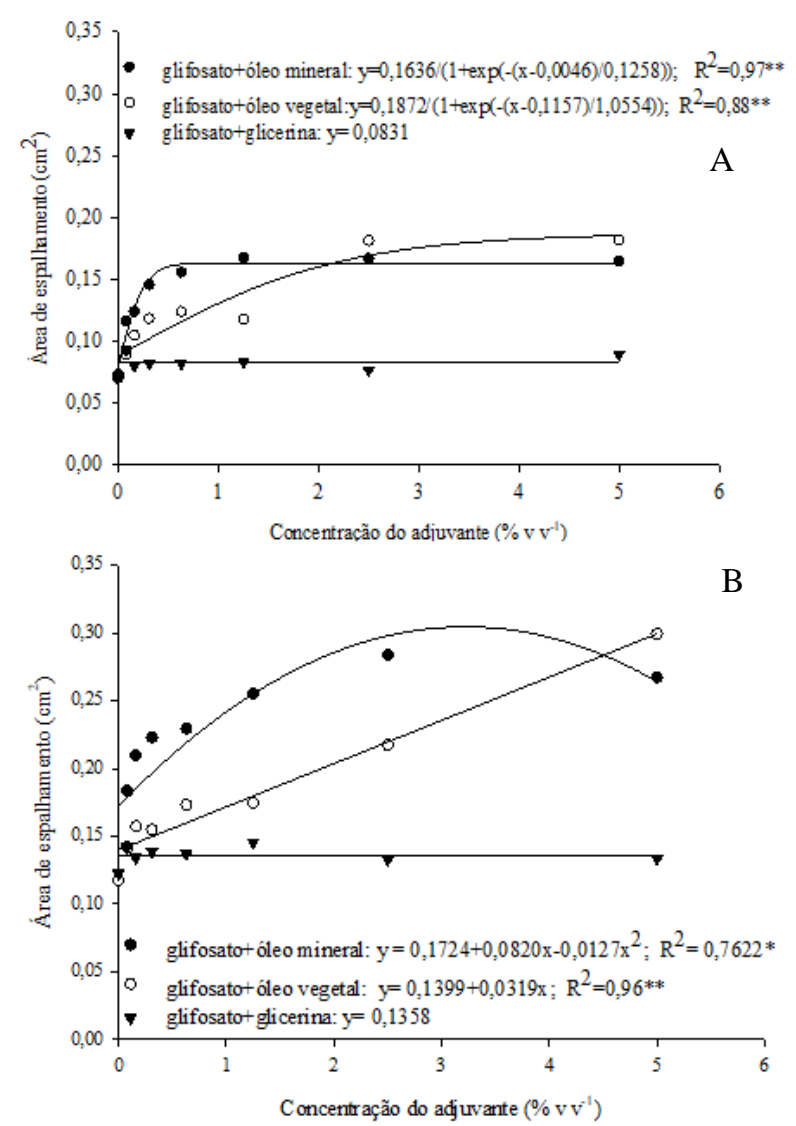

Figura 2. Área de espalhamento de gotas na face abaxial (A) e adaxial (B) da folha de D. insularis das soluções de glifosato com diferentes concentrações de adjuvantes.

Na Figura 3, estão apresentados os valores da área de espalhamento das gotas na superfície abaxial e adaxial da folha de $D$. insularis das soluções de glufosinato com concentrações de adjuvantes.

$\mathrm{Na}$ face abaxial, os dados de espalhamento de gotas do glufosinato + óleo vegetal apresentaram comportamento linear, sendo que ocorreu espalhamento de $0,0165 \mathrm{~cm}^{2}$ na folha a cada aumento da concentração do adjuvante (Figura 3A). Entretanto, o glufosinato + óleo mineral $(1,5 \%)$ e glufosinato + glicerina $(0,2 \%)$ apresentaram os maiores espalhamentos, sendo 48,6 e $25,6 \%$, respectivamente superior em relação ao obtida pela solução do herbicida sem adjuvante.

Para a face adaxial (Figura 3B), os maiores espalhamentos foram verificados para as soluções de glufosinato+óleo mineral $(0,6 \%)$ e glufosinato+óleo vegetal $(0,3 \%)$ que proporcionaram 32,5 e $43,8 \%$, respectivamente, mais espalhamento do que o herbicida sem adjuvante.

Ressalta-se ainda que a diferença de espalhamento entre as faces foliares da $D$. insularis pode ocorrer devido à desuniformidade da distribuição de ceras, depositadas em maior quantidade na superfície adaxial das folhas (Hess; Falk, 1990), podendo reduzir o espalhamento das gotas.
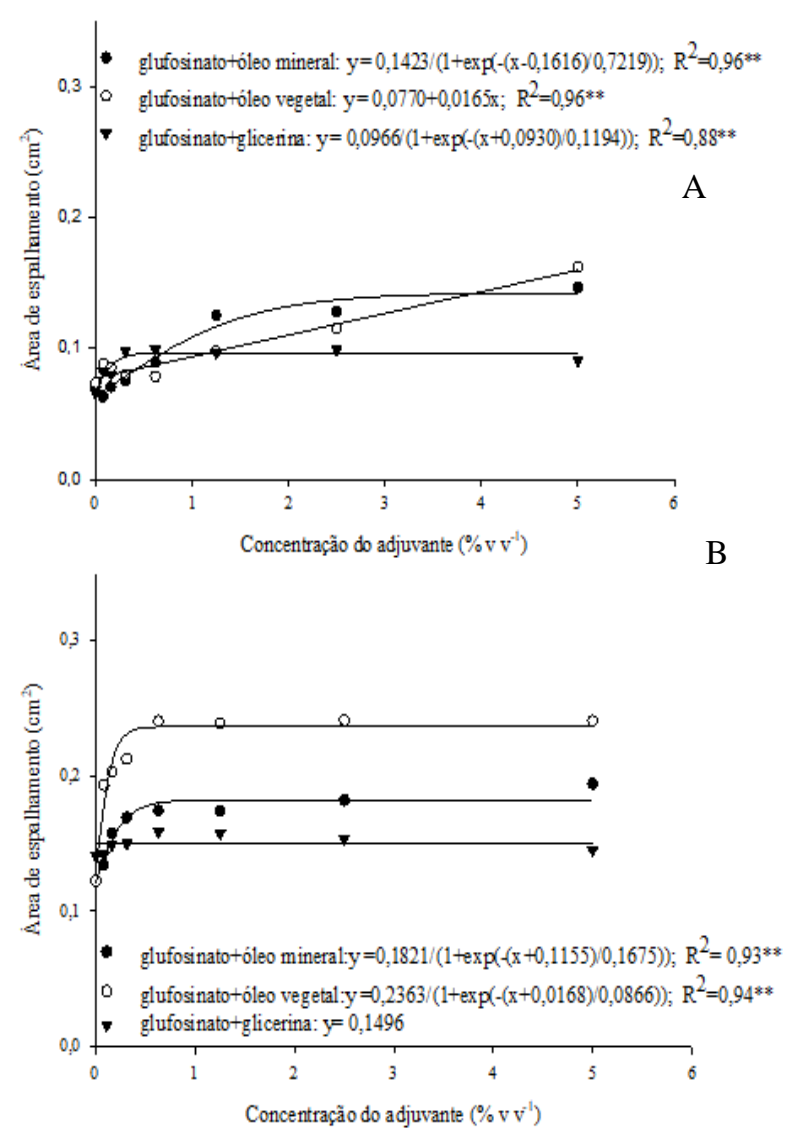

Figura 3. Área de espalhamento de gotas na face abaxial (A) e adaxial (B) da folha de $D$. insularis das soluções de glufosinato com diferentes concentrações de adjuvantes.

Os dados de glicerina não apresentaram ajuste, entretanto, houve menor eficiência em promover espalhamento da gota, em comparação ao óleo mineral e vegetal. (Baio et al. 2015), verificaram que a glicerina (Hygrogem $^{\circledR}$ - 0,5) foi eficiente em reduzir a tensão superficial da gota, entretanto, o espalhamento em folhas soja proporcionado pela glicerina foi 67,0 e $70,4 \%$ superior do que o espalhamento do óleo vegetal $\left(\right.$ Áureo $^{\circledR}$ - 0,25) e óleo mineral (Nimbus ${ }^{\circledR}$ - 0,5), respectivamente. Destaca-se que este trabalho foi realizado sem o uso de produtos químicos, o que poderia alterar os resultados.

Existem poucas informações na literatura sobre a eficiência de adjuvantes no espalhamento de gotas em superfícies foliares principalmente com o herbicida 
glufosinato. Contudo, a redução da tensão superficial contribui para o aumento da área de espalhamento nas folhas (Mendonça et al., 1999), bem como pode aumentar a absorção do e a eficiência de controle, principalmente de herbicidas de ação de contato.

Maciel et al. (2011) verificaram que ao adicionar fertilizante foliar (Triunfo $^{\circledR}$ ), anti-deriva (Grap Super Gun ${ }^{\circledR}$ ) e óleo mineral (Assist ${ }^{\circledR}$ ), todos na concentração $0,035 \% \mathrm{v} \mathrm{v}^{-1}$, promoveram incremento no controle do $D$. insularis para o glifosato $\left(2,25 \mathrm{~L} \mathrm{ha}^{-1}\right)$. (Velini et al. 2000) observaram que a adição de $0,1 \%$ do adjuvante Aterbane ${ }^{\circledR}$ permitiu reduzir a dose do herbicida em 10 $\%$ mantendo o mesmo nível de controle $(90 \%)$ da Brachiaria decumbens e Panicum maximum.

Para o glufosinato, (Melo et al.,2012) verificou que o glufosinato $\left(600 \mathrm{~g} \mathrm{ha}^{-1}\right)+$ espalhante adesivo $\left(\right.$ Agral $^{\circledR}$ - 0,2\%) não foi eficiente para o controle de $D$. insularis. Entretanto, o uso de tecnologias de aplicação, tais como o uso de adjuvantes, possibilitariam o aumento da eficácia de controle.

De acordo com os resultados obtidos, observou-se que os adjuvantes foram eficientes em reduzir a tensão superficial das soluções com os herbicidas, com exceção para a glicerina adicionada ao glifosato. A eficiência no espalhamento de gotas nas folhas de $D$. insularis foi dependente do herbicida, da face foliar, bem como da concentração dos adjuvantes avaliados.

\section{Conclusão}

Apenas o glifosato nas diferentes concentrações de glicerina não promoveu redução da tensão superficial. $\mathrm{O}$ glifosato + óleo mineral $(0,6 \%)$ e o glufosinato + óleo mineral $(1,5 \%)$ foram os mais eficientes no espalhamento na face abaxial. Enquanto que, glifosato + óleo mineral $(3,2 \%)$ e o glufosinato + óleo vegetal $(0,3 \%)$ foram os mais eficientes no espalhamento na face adaxial da folha de $D$. insularis.

\section{Referências Bibliográficas}

Baio, F. H. R.; Gabriel, R. R. F.; Camolese, H. S., 2015. Alteração das propriedades físico-químicas na aplicação contendo adjuvantes. Brazilian Journal of Biosystems Engineering, 9, 151-161.

Costa, N. V.; Modolon, T. A.; Pisatto, M.; Broetto, L.; Mezzalira, E. J., 2014. Tensão superficial e área de espalhamento de gotas de soluções com herbicidas e adjuvantes em folhas de Conyza canadensis. Scientia Agraria Paranaensis, 13, 161-170.

Cunha, J. P. A. R.; Alves, G. S., 2009. Características físicoquímicas de soluções aquosas com adjuvantes de uso agrícola. Interciência, 34, 655-659.
Cunha, J. P. A. R.; Alves, G. S.; Reis, E. F., 2010. Efeito da temperatura nas características físico-químicas de soluções aquosas com adjuvantes de uso agrícola. Planta Daninha,28, 665-672.

Galon, L.; Concenço, G.; Ferreira, E. A.; Silva, A. F.; Ferreira, F. A.; Noldin, J. A. Freitas, M. A. M., 2007. Competição entre plantas de arroz e biótipos de capim-arroz (echinochloa spp.) resistente e suscetível ao quinclorac. Planta Daninha, 27, 701709 .

Hess, F. D.; Falk, R. H., 1990. Herbicide deposition on leaf surfaces. Weed Science, 38, 280-288.

Maciel, C. D. G., Moraes, D. W.; Balan, M. G., 2011. Associação de adjuvantes com herbicidas na dessecação e no controle em pós-emergência de plantas daninhas na cultura do trigo. Revista Brasileira de Herbicidas, 10, 243-256.

Machado, A. F. L.; Ferreira, L. R.; Ferreira, F. A.; Fialho, C. M. T.; Tuffi, S. L. D.; Machado, M .S., 2006. Análise de crescimento de Digitaria insularis. Planta Daninha, 24, 641-64.

Machado, A. F. L.; Meira, R. M. S.; Ferreira, L. R.; Ferreira, F. A.; Tuffi, S. L. D.; Fialho, C. M. T.; Machado, M. S., 2008. Caracterização anatômica de folha, colmo e rizoma de Digitaria insularis. Planta Daninha, 26 1-8.

Mendonça, C. G.; Velini, E. D.; Martins, D.; Mendonça, C. G., 1999. Efeitos de surfatantes sobre a tensão superficial e a área de molhamento de soluções de glyphosate sobre folhas de tiririca. Planta Daninha, 17, 355-365.

Mendonça, C. G.; Raetano, C. G.; Mendonça, C. G., 2007. Tensão superficial estática de soluções aquosas com óleos minerais e vegetais utilizados na agricultura. Engenharia Agrícola, 16-23.

Melo, S. C. M.; Rosa, L. E.; Brunharo, C. A. C. G.; Nicolai, M.; Christoffoleti, P. J., 2012. Alternativas para o controle de capim-amargoso (Digitaria insularis) resistente ao glyphosate. Revista Brasileira de Herbicidas, 11, 195-203.

Moreira, M .S; Nicolai, M.; Carvalho, S. J. P.; Christoffoleti, P. J., 2007. Resistência de Conyza canadensis e C. bonariensis ao herbicida glyphosate. Planta Daninha, 25, 157-164.

Procópio, S.O.; Pires, F. R.; Menezes, C. C. E.; Barroso, A. L. L.; Moraes, R. V.; Silva, M. V. V.; Queiroz, R. G.; Carmo, M. L., 2006. Efeitos de dessecantes no controle de plantas daninhas na cultura da soja. Planta Daninha, 24, 193-197.

Velini, E. D.; Martins, D.; Silva, M. A. S., 2000. Efeito de concentrações de espalhante adesionante e doses de glyphosate no controle de Brachiaria decumbens e Panicum maximum. Planta Daninha, 18, 349-366.

Vidal, R. A.; Spader, V.; Fleck, N. G.; Merotto Junior, A., 2004. Nível de dano econômico de brachiaria plantaginea na cultura de milho irrigado. Planta Daninha, 22, 63-69.

Voll, E.; Gazziero, D. L. P.; Brighenti, A. A. M.; Adegas, F. S., 2002. Competição relativa de espécies de plantas daninhas com dois cultivares de soja. Planta Daninha, 20, 17-24. 